\title{
Transient quantum transport in double-dot Aharonov-Bohm interferometers
}

\author{
Matisse Wei-Yuan Tu and Wei-Min Zhand* \\ Department of Physics, National Cheng Kung University, Tainan 70101, Taiwan \\ JinShuang Jin \\ Department of Physics, Hangzhou Normal University, Hangzhou 310036, China \\ O. Entin-Wohlmant and A. Aharony \\ Physics Department, Ben Gurion University, Beer Sheva 84105, Israel
}

(Dated: May 1, 2012)

\begin{abstract}
Real-time nonequilibrium quantum dynamics of electrons in double-dot Aharonov-Bohm (AB) interferometers is studied using an exact solution of the master equation. The building of the coherence between the two electronic paths shows up via the time-dependent amplitude of the $\mathrm{AB}$ oscillations in the transient transport current, and can be enhanced by varying the applied bias on the leads, the on-site energy difference between the dots and the asymmetry of the coupling of the dots to the leads. The transient oscillations of the transport current do not obey phase rigidity. The circulating current has an anti-symmetric AB oscillation in the flux. The non-degeneracy of the on-site energies and the finite bias cause the occupation in each dot to have an arbitrary flux dependence as the coupling asymmetry is varied.

PACS numbers: 73.23.-b, 73.63.-b
\end{abstract}

\section{INTRODUCTION}

Coherence of electronic transport through mesoscopic junctions has been studied intensively in nanoelectronic systems. In particular, the interference of electron waves has been visualized in Aharonov-Bohm (AB) interferometers via the $\mathrm{AB}$ oscillations of the conductance of a ring placed between two leads. Following the electron injection from the leads into the ring, the electrons undergo a nonequilibrium transport process before a steady interference pattern is reached. While the steady-state AB interference has been largely explored in the literature, the real-time dynamics of electronic transport in AB interferometers has not yet been fully understood. In this paper we study this dynamics in double-quantum-dot $\mathrm{AB}$ interferometers addressing the transient $\mathrm{AB}$ interference under various tunable parameters of the system.

The study of the wavy nature of electronic transmissions has been mainly focused on the complex amplitudes of the transmitted electrons in the scattering approach $\stackrel{1,2}{*}$ The archetype model contains a single quantum dot sitting on one of the two arms of the $\mathrm{AB}$ ring. A quantum point contact (QPC) placed nearby the quantum dot has been used to study the effect of a which-path detection ${ }^{3}$ A single-dot $\mathrm{AB}$ interferometer has been realized in a closed geometry ${ }^{4}$ and also in an open one $\frac{5}{-}$ Phase rigidity in a two-terminal geometry has been experimentally discovered ${ }^{4,6}$ and theoretically explained ${ }^{7}$ The effect of electron-electron interactions on transport through AB interferometers has also been explored ${ }^{8.9} \mathrm{~A}$ review on the early progress can be found in Ref. 10]. Extracting the transmission phase from the AB oscillations is another main issue. The way continuous phase shifts (as opposed to phase rigidity) of the AB oscillations can be induced by breaking the unitarity of the scatter- ing matrix and the way such phase shifts depend on the properties of electron losses have been investigated. 11,12 Likewise, ways of extracting both the amplitude and the phase of the intrinsic transmission amplitude from the measured conductance without opening the interferometer have been suggested 13 The studies of AB interferometers with two quantum dots placed on the two arms of the ring have been focused on different issues, such as the flux-dependent level attraction, 14 the effect of intradot Coulomb interactions, $\stackrel{15,16}{=}$ inelastic scattering with phonons $\frac{17}{17}$ as well as extracting transmission phases from the current measurements using QPC placed next to the one of the quantum dots 18

The above investigations are concentrated mainly on steady-state properties of quantum-dot AB interferometers. In this paper we consider the transient transport behaviors in this system. We consider a double-quantumdot $\mathrm{AB}$ interferometer as sketched in Fig. 1, where a single active charge state on each dot is assumed and electron-electron interactions are ignored. In a recent work, $\frac{19}{1}$ some of us have studied the electron dynamics in this system under the condition of identical onsite energies of the dots and symmetric couplings to the leads. In that study, a phase localization phenomenon has been found. In the present paper, we systematically explore the general transient transport dynamics with non-identical on-site energies on the dots and asymmetric couplings to the leads. In particular, besides the search for the dynamical flux dependences of the transient net current, we also examine the flux dependence of the transient electronic occupation in each dot and the transient circulating current. The electronic occupation on each dot can be measured in experiments and contains rich information about the transport processes. The relatively large circulating current at zero or small bias may 
provide new insights into electron coherence during the transport.

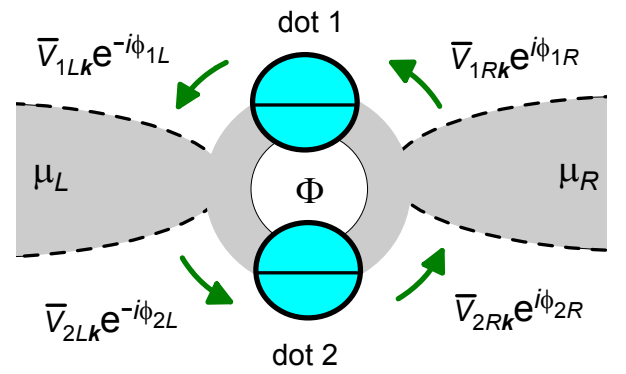

FIG. 1: A schematic sketch of the system: the AharonovBohm interferometer, consisting of two single-level dots, is connected to a source and a drain set at different chemical potentials, $\mu_{L}$ and $\mu_{R}$, respectively. The interferometer is threaded by a magnetic flux $\Phi$ measured in units of the flux quantum $\Phi_{0}=h c / e$.

Here is a summary of the main results we obtain. By setting the two electronic leads (as the reservoirs) at thermal equilibrium initially with no excess electrons on the double dot, we monitor the time evolutions of the electronic charge occupation, the transport net current and the circulating current. When the two on-site energies on the dots are identical (namely the double dot is degenerate), regardless of the coupling asymmetry to the leads, we find that the total electronic occupation on the double dot and the net current are always symmetric in the flux, while the occupation difference between the two dots and the circulating current are always anti-symmetric in it. We also find that the times needed for the total occupation to reach its steady-state values are much longer near zero flux, compared with the case where the flux value is away from zero. By breaking the degeneracy of the double dot, the net current is allowed to break phase rigidity transiently at any bias. The flux dependence of the total occupation number changes arbitrarily as the the coupling asymmetry is varied at finite biases. The non-degenerate double dot coupled asymmetrically to the leads also drives the circulating current slightly away from an anti-symmetric flux dependence immediately after the current is switched on, but it then quickly becomes completely anti-symmetric in the flux.

The rest of the paper is organized as follows. In Sec. II we outline the basic formalism describing the nonequilibrium electronic dynamics for nanoelectronic devices in general, and for the double-dot $\mathrm{AB}$ interferometer in particular. In Sec. III we present analytical expressions for the electronic occupations, the transient net current and the circulating current. In Sec. IV we consider the steady-state limit, reproduce known results for this system and compare them with ours. In Sec. V we numerically demonstrate the transient flux dependence of the electronic occupations and currents. Finally, conclusions are drawn in Sec. VI.

\section{BASIC FORMALISM}

In this section we give a brief introduction to the nonequilibrium quantum theory that can describe transient quantum transport and quantum coherence in nanoelectronic systems 20,21 and then apply it to the doublequantum-dot $\mathrm{AB}$ interferometer considered in this paper.

The Hamiltonian of the prototypical nanoelectronic system we consider can be written as

$$
\mathcal{H}=\mathcal{H}_{\mathrm{s}}+\mathcal{H}_{\mathrm{E}}+\mathcal{H}_{\mathrm{T}}
$$

where $\mathcal{H}_{\mathrm{s}}=\sum_{i j} E_{i j} a_{i}^{\dagger} a_{j}$ is the Hamiltonian of the central system with $i, j$ labeling the electronic levels in the dots, $\mathcal{H}_{\mathrm{E}}=\sum_{\alpha \boldsymbol{k}} \epsilon_{\alpha \boldsymbol{k}} c_{\alpha \boldsymbol{k}}^{\dagger} c_{\alpha \boldsymbol{k}}$ is the lead Hamiltonian with $\alpha$ labeling the leads and $\boldsymbol{k}$ denoting the states in the leads, and $\mathcal{H}_{\mathrm{T}}=\sum_{i \alpha \boldsymbol{k}}\left[V_{i \alpha \boldsymbol{k}} c_{\alpha \boldsymbol{k}}^{\dagger} a_{i}+\right.$ H.c. $]$ describing the tunneling between the dots and the leads. Here $a_{i}^{\dagger}\left(a_{i}\right)$ and $c_{\alpha \boldsymbol{k}}^{\dagger}\left(c_{\alpha \boldsymbol{k}}\right)$ are the electron creation (annihilation) operators for electronic levels $i$ and $\boldsymbol{k}$ in the dots and in lead $\alpha$, respectively. $E_{i i}=E_{i}$ is the energy of level $i, E_{i j}(i \neq j)$ is the tunneling amplitude between the different levels in the dots, and $V_{i \alpha k}$ is the tunneling amplitude between the dots and the leads. Electron-electron interactions are ignored.

Since the central system is open to the electron reservoirs (via the leads), its nonequilibrium dynamics is naturally described by the reduced density matrix $\rho(t)$ which is defined by tracing over the states of the leads,

$$
\rho(t)=\operatorname{tr}_{\mathrm{E}} \rho_{\mathrm{tot}}(t)=\operatorname{tr}_{\mathrm{E}}\left[e^{-i \mathcal{H}\left(t-t_{0}\right)} \rho_{\mathrm{tot}}\left(t_{0}\right) e^{i \mathcal{H}\left(t-t_{0}\right)}\right],
$$

where $\rho_{\text {tot }}(t)$ is the total density matrix of the central system plus the leads. Electronic occupations on the discrete electronic states in the dots can be read from $\rho(t)$. The electronic transport through the central system is characterized by the currents flowing from the leads into the dots, defined by $I_{\alpha}=-e \frac{d}{d t} \sum_{\boldsymbol{k} \in \alpha} \operatorname{tr}_{t o t}\left[c_{\alpha \boldsymbol{k}}^{\dagger} c_{\alpha \boldsymbol{k}} \rho_{\text {tot }}(t)\right]$ for lead $\alpha$. This can be further decomposed into separate contributions through each dot:

$$
I_{\alpha}=\sum_{i} I_{i \alpha}, \quad I_{i \alpha}=i e \sum_{\boldsymbol{k} \in \alpha} \operatorname{tr}_{\mathrm{tot}}\left[V_{i \alpha \boldsymbol{k}} c_{\alpha \boldsymbol{k}}^{\dagger} a_{i} \rho_{\mathrm{tot}}(t)-\text { H.c. }\right] \text {. }
$$

In Eqs. (2) and (3), $\operatorname{tr}_{E}$ and $\operatorname{tr}_{\text {tot }}$ denote the traces over the states of the leads and the total system, respectively. Throughout the paper, we use units in which $\hbar=1$.

As usual, we assume 22 that the central dot system is initially decoupled from the leads, and the leads are initially at thermal equilibrium with the chemical potential $\mu_{\alpha}$ and inverse temperature $\beta=1 / k_{B} T$ for lead $\alpha$, whose Fermi distribution function is given by $f_{\alpha}(\epsilon)=$ $1 /\left[e^{\beta\left(\epsilon-\mu_{\alpha}\right)}+1\right]$. Then the exact equations governing the time evolution of the reduced density matrix and the transient currents are 20,21

$$
\begin{aligned}
& \frac{d}{d t} \rho(t)=-i\left[\mathcal{H}_{\mathrm{s}}, \rho(t)\right]+\sum_{i \alpha}\left[\mathcal{L}_{i \alpha}^{+}(t)+\mathcal{L}_{i \alpha}^{-}(t)\right] \rho(t), \\
& I_{i \alpha}(t)=e \operatorname{tr}_{\mathrm{s}}\left[\mathcal{L}_{i \alpha}^{+}(t)\right]=-e \operatorname{tr}_{\mathrm{s}}\left[\mathcal{L}_{i \alpha}^{-}(t) \rho(t)\right],
\end{aligned}
$$


where the superoperators $\mathcal{L}_{i \alpha}^{ \pm}(t)$ are expressed explicitly by

$$
\begin{aligned}
\mathcal{L}_{i \alpha}^{+}(t) \rho(t)=- & \sum_{j}\left\{\boldsymbol{\lambda}_{\alpha i j}(t)\left[a_{i}^{\dagger} a_{j} \rho(t)+a_{i}^{\dagger} \rho(t) a_{j}\right]\right. \\
& \left.+\boldsymbol{\kappa}_{\alpha i j}(t) a_{i}^{\dagger} a_{j} \rho(t)+\text { H.c. }\right\}, \\
\mathcal{L}_{i \alpha}^{-}(t) \rho(t)= & \sum_{j}\left\{\boldsymbol{\lambda}_{\alpha i j}(t)\left[a_{j} \rho(t) a_{i}^{\dagger}+\rho(t) a_{j} a_{i}^{\dagger}\right]\right. \\
& \left.+\boldsymbol{\kappa}_{\alpha i j}(t) a_{j} \rho(t) a_{i}^{\dagger}+\text { H.c. }\right\},
\end{aligned}
$$

and $\operatorname{tr}_{s}$ is the trace over the states of the dots. The first term on the right hand side of Eq. (4a) is the renormalized Liouville operator of the central dot system. The second and the third terms, expressed in terms of the superoperators, are non-unitary. The non-unitarity is induced by electronic dissipation and fluctuation processes due to the couplings of the central dot system to the electronic reservoirs. The transient transport current is determined from the non-unitary dynamics, as shown by Eq. (4b). Equations (4) and (5) form the basis of the nonequilibrium description of quantum coherence and quantum transport in mesoscopic systems.

The time-dependent dissipation and fluctuation coefficients in Eqs. (5), $\boldsymbol{\kappa}_{\alpha}(t)$ and $\boldsymbol{\lambda}_{\alpha}(t)$, are explicitly determined by the nonequilibrium retarded and correlation Green functions of the dot system, denoted here by $\boldsymbol{u}(t)$ and $\boldsymbol{v}(t) \stackrel{21}{\Perp}$ via the relations

$\boldsymbol{\kappa}_{\alpha}(t)=\int_{t_{0}}^{t} d \tau \boldsymbol{g}_{\alpha}(t, \tau) \boldsymbol{u}(\tau) \boldsymbol{u}^{-1}(t)$,
$\boldsymbol{\lambda}_{\alpha}(t)=\int_{t_{0}}^{t} d \tau\left\{\boldsymbol{g}_{\alpha}(t, \tau) \boldsymbol{v}(\tau)-\widetilde{\boldsymbol{g}}_{\alpha}(t, \tau) \overline{\boldsymbol{u}}(\tau)\right\}-\boldsymbol{\kappa}_{\alpha}(t) \boldsymbol{v}(t)$.

The nonequilibrium retarded and correlation Green functions of the dot system obey the following dissipationfluctuation integrodifferential equations of motion

$$
\begin{gathered}
\frac{d}{d t} \boldsymbol{u}(\tau)+i \boldsymbol{E} \boldsymbol{u}(\tau)+\int_{t_{0}}^{\tau} d \tau^{\prime} \boldsymbol{g}\left(\tau-\tau^{\prime}\right) \boldsymbol{u}\left(\tau^{\prime}\right)=0 \\
\frac{d}{d t} \boldsymbol{v}(\tau)+i \boldsymbol{E} \boldsymbol{v}(\tau)+\int_{t_{0}}^{\tau} d \tau^{\prime} \boldsymbol{g}\left(\tau-\tau^{\prime}\right) \boldsymbol{v}\left(\tau^{\prime}\right) \\
=\int_{t_{0}}^{t} d \tau^{\prime} \widetilde{\boldsymbol{g}}\left(\tau-\tau^{\prime}\right) \overline{\boldsymbol{u}}\left(\tau^{\prime}\right)
\end{gathered}
$$

subject to the conditions $\boldsymbol{u}\left(t_{0}\right)=I, \boldsymbol{v}\left(t_{0}\right)=0$ with $t_{0} \leq$ $\tau \leq t$, and $\overline{\boldsymbol{u}}(\tau)=\boldsymbol{u}^{\dagger}\left(t-\tau+t_{0}\right)$ is the advanced Green function. Here $\boldsymbol{E}$ is the on-site energy matrix of the dot system, $\boldsymbol{g}=\sum_{\alpha} \boldsymbol{g}_{\alpha}$ and $\tilde{\boldsymbol{g}}=\sum_{\alpha} \widetilde{\boldsymbol{g}}_{\alpha}$ are the self-energy corrections due to the coupling to the leads:

$$
\begin{aligned}
& \boldsymbol{g}_{\alpha}(\tau)=\int \frac{d \omega}{2 \pi} \boldsymbol{\Gamma}_{\alpha}(\omega) e^{-i \omega \tau}, \\
& \widetilde{\boldsymbol{g}}_{\alpha}(\tau)=\int \frac{d \omega}{2 \pi} f_{\alpha}(\omega) \boldsymbol{\Gamma}_{\alpha}(\omega) e^{-i \omega \tau} .
\end{aligned}
$$

The spectral density $\boldsymbol{\Gamma}_{\alpha i j}(\omega)=2 \pi \sum_{\boldsymbol{k} \in \alpha} V_{i \alpha \boldsymbol{k}}^{*} V_{j \alpha \boldsymbol{k}} \delta(\omega-$ $\left.\epsilon_{\alpha \boldsymbol{k}}\right)$ summarizes all the non-Markovian memory effects of the electron reservoirs on the dot system.

The correlation Green function $\boldsymbol{v}(t)$, Eq. (7b), has a general solution in terms of the retarded Green function $\boldsymbol{u}(\tau)$

$$
\boldsymbol{v}(\tau)=\int_{t_{0}}^{\tau} d \tau_{1} \int_{t_{0}}^{t} d \tau_{2} \boldsymbol{u}\left(\tau-\tau_{1}+t_{0}\right) \widetilde{\boldsymbol{g}}\left(\tau_{1}-\tau_{2}\right) \overline{\boldsymbol{u}}\left(\tau_{2}\right) .
$$

From the master equation, Eq. (4a), it is easy to find the single-particle reduced density matrix in terms of $\boldsymbol{u}(t)$ and $\boldsymbol{v}(t)$ :

$$
\rho_{i j}^{(1)}(t) \equiv \operatorname{tr}\left[a_{j}^{\dagger} a_{i} \rho(t)\right]=u_{i i^{\prime}}(t) \rho_{i^{\prime} j^{\prime}}^{(1)}\left(t_{0}\right) u_{j^{\prime} j}^{\dagger}(t)+v_{i j}(t),
$$

where $\rho_{i j}^{(1)}\left(t_{0}\right)$ is the initial single-particle reduced density matrix of the dots. The currents Eq. (4b) can then be explicitly expressed as 21

$$
\begin{aligned}
& I_{i \alpha}(t)=-2 e \operatorname{Re} \int_{t_{0}}^{t} d \tau\left\{\boldsymbol{g}_{\alpha}(t-\tau) \boldsymbol{v}(\tau)-\widetilde{\boldsymbol{g}}_{\alpha}(t-\tau) \overline{\boldsymbol{u}}(\tau)\right. \\
&\left.+\boldsymbol{g}_{\alpha}(t-\tau) \boldsymbol{u}(\tau) \boldsymbol{\rho}^{(1)}\left(t_{0}\right) \overline{\boldsymbol{u}}^{\dagger}(t)\right\}_{i i} .
\end{aligned}
$$

This expression is consistent with the result obtained from the Keldysh Green function technique, except that the initial state dependence [the third term in Eq. (10)] is usually ignored in most of the Green function treatments ${ }^{23}$ (see the explicit derivation given in Ref. [21]). If the dot system is initially empty, namely, $\rho_{i j}^{(1)}\left(t_{0}\right)=0$, the transient electronic occupations and currents can be further simplified:

$\rho_{i j}^{(1)}(t)=v_{i j}(t)$,
$I_{i \alpha}(t)=-2 e \operatorname{Re} \int_{t_{0}}^{t} d \tau\left\{\boldsymbol{g}_{\alpha}(t-\tau) \boldsymbol{v}(\tau)-\widetilde{\boldsymbol{g}}_{\alpha}(t-\tau) \overline{\boldsymbol{u}}(\tau)\right\}_{i i}$.

Thus, solving Eq.(7a) and using Eq. (9), we can obtain the full information of the transient quantum transport dynamics.

To be specific, we consider in this paper a doublequantum-dot $\mathrm{AB}$ interferometer schematically plotted in Fig. 1 where each of the quantum dots has a single active electronic state. Then the energy matrix $\boldsymbol{E}$ in Eq. (7) becomes a $2 \times 2$ matrix. We also do not consider the inter-dot tunnel coupling, namely, $E_{12}=$ $E_{21}=0$. The $\mathrm{AB}$ magnetic flux is embedded in the tunneling amplitudes between the leads and the dots: $V_{j L \boldsymbol{k}}=\bar{V}_{j L \boldsymbol{k}} e^{-i \phi_{j L}}$ and $V_{j R \boldsymbol{k}}=\bar{V}_{j R \boldsymbol{k}} e^{i \phi_{j R}}$ with the relation $\phi_{1 L}-\phi_{2 L}+\phi_{1 R}-\phi_{2 R}=\phi \equiv 2 \pi \Phi / \Phi_{0}$, and $\Phi_{0}=h c / e$ is the flux quantum. Thus the spectral density involving explicitly the threading magnetic flux is given by

$$
\boldsymbol{\Gamma}_{\alpha i j}(\omega)=2 \pi \sum_{\boldsymbol{k} \in \alpha} \bar{V}_{i \alpha \boldsymbol{k}} \bar{V}_{j \alpha \boldsymbol{k}} e^{ \pm i\left(\phi_{i \alpha}-\phi_{j \alpha}\right)} \delta\left(\omega-\epsilon_{\alpha \boldsymbol{k}}\right),
$$


where the $+(-)$ sign is for $\alpha=L(R)$. With the above basic formulation, we are able to explore the nonequilibrium electronic dynamics in this nanoscale $\mathrm{AB}$ interferometer.

\section{ANALYTICAL SOLUTIONS}

We exploit in our calculations the ubiquitously-used wide-band approximation, in which the spectral density is assumed to be frequency independent. In general, the magnetic phase can be characterized by two variables, the magnetic flux threading the ring, $\phi=\phi_{L}+\phi_{R}$, and the difference, i.e. the gauge degree of freedom, $\chi=$ $\frac{\phi_{L}-\phi_{R}}{2}$, where $\phi_{\alpha}=\phi_{1 \alpha}-\phi_{2 \alpha}$. Correspondingly, the spectral density is reduced to $\boldsymbol{\Gamma}_{\alpha}=\Gamma_{\alpha}\left(\begin{array}{cc}1 & e^{ \pm i \phi_{\alpha}} \\ e^{\mp i \phi_{\alpha}} & 1\end{array}\right)$, where the upper (lower) sign is for $\alpha=L(R)$. The timedependent self-energy correction to the retarded Green function of the electron in the double dot is given by

$$
\boldsymbol{g}(\tau)=\delta(\tau)\left(\begin{array}{cc}
\Gamma & e^{i \chi} \Gamma_{\phi}^{+} \\
e^{-i \chi} \Gamma_{\phi}^{-} & \Gamma
\end{array}\right)
$$

with $\Gamma_{\phi}^{ \pm}=[\Gamma \cos (\phi / 2) \pm i \delta \Gamma \sin (\phi / 2)]$. Here $\Gamma=\Gamma_{L}+\Gamma_{R}$ and $\delta \Gamma=\Gamma_{L}-\Gamma_{R}$ characterize the strength and the asymmetry of the coupling to the leads, respectively.

Even for the most general case of a non-degenerate double dot asymmetrically coupled to the leads, the solution of Eq. (7a) can be found analytically (taking $t_{0}=0$ ):

$$
\boldsymbol{u}(\tau)=u_{0}(\tau) \sigma_{0}-u_{p}(\tau) \hat{\vec{p}}(\phi, \chi) \cdot \vec{\sigma} .
$$

Here $\vec{\sigma}=\left(\sigma_{+}, \sigma_{-}, \sigma_{z}\right)$ is the vector of the three Pauli matrices, and $\sigma_{0}=I$ (the identity operator). We have introduced a flux-dependent and gauge-dependent polarization vector $\vec{p}(\phi, \chi) \equiv$ $\left(p_{-}(\phi, \chi), p_{+}(\phi, \chi), p_{z}(\phi, \chi)\right)=\left(\frac{1}{2} e^{i \chi} \Gamma_{\phi}^{+}, \frac{1}{2} e^{-i \chi} \Gamma_{\phi}^{-}, i \delta E\right)$ containing all the information on the gauge dependence, the flux dependence and the dependence on the asymmetry of the couplings, with $\delta E=E_{1}-E_{2}$ characterizing the non-degeneracy of the double dot on-site energies. Here $\hat{\vec{p}}(\phi, \chi)=\vec{p}(\phi, \chi) / \Gamma_{\phi}$ and $\Gamma_{\phi}=\sqrt{\Gamma^{2} \cos ^{2}(\phi / 2)+\delta \Gamma^{2} \sin ^{2}(\phi / 2)-\delta E^{2}}$ which is gauge independent. Without loss of generality, we set $E=\frac{E_{1}+E_{2}}{2}=0$, as an energy reference. Then the functions $u_{0}(\tau)$ and $u_{p}(\tau)$ in Eq. (15) are given by

$$
u_{0, p}(\tau)=\frac{1}{2}\left[e^{-\gamma_{\phi}^{-} \tau} \pm e^{-\gamma_{\phi}^{+} \tau}\right],
$$

with $\gamma_{\phi}^{ \pm}=\frac{1}{2}\left(\Gamma \pm \Gamma_{\phi}\right)$, which are also gauge independent. Substituting Eq. (15) into Eq. (9), we obtain the correlation Green function

$$
\boldsymbol{v}(t)=\int \frac{d \omega}{2 \pi} \boldsymbol{u}(t, \omega) \sum_{\alpha} f_{\alpha}(\omega) \boldsymbol{\Gamma}_{\alpha} \boldsymbol{u}^{\dagger}(t, \omega),
$$

where $\boldsymbol{u}(t, \omega)=u_{0}(t, \omega) \sigma_{0}-u_{p}(t, \omega) \hat{\vec{p}}(\phi, \chi) \cdot \vec{\sigma}$ and

$$
u_{0, p}(t, \omega)=\frac{1}{2}\left[\frac{e^{\left(i \omega-\gamma_{\phi}^{-}\right) t}-1}{i \omega-\gamma_{\phi}^{-}} \pm \frac{e^{\left(i \omega+\gamma_{\phi}^{+}\right) t}-1}{i \omega-\gamma_{\phi}^{+}}\right] .
$$

The gauge degree of freedom parameterized by $\chi$ appears explicitly in the off-diagonal matrix elements of the retarded and the correlation Green functions $\boldsymbol{u}(t)$ and $\boldsymbol{v}(t)$. However, the physical observables, calculated from $\boldsymbol{u}(t)$ and $\boldsymbol{v}(t)$, do not depend on $\chi$, ensuring the gauge invariance of our calculations.

Explicitly, the electronic occupation on each dot is given by the diagonal matrix element of $\boldsymbol{v}(t)$, see Eq. (12a). The total occupation number $N(t)=n_{1}(t)+$ $n_{2}(t)$, where $n_{i}(t)=v_{i i}(t)$, can be expressed explicitly as

$$
\begin{aligned}
& N(t)=\int \frac{d \omega}{2 \pi} f_{+}(\omega)\left\{\Gamma\left[\left|u_{0}(t, \omega)\right|^{2}+\left(\Gamma_{\phi}^{2}+2 \delta E^{2}\right)\left|\frac{u_{p}(t, \omega)}{\Gamma_{\phi}}\right|^{2}\right]-2\left(\Gamma_{\phi}^{2}+\delta E^{2}\right) \operatorname{Re}\left[\frac{u_{0}^{*}(t, \omega) u_{p}(t, \omega)}{\Gamma_{\phi}}\right]\right\} \\
& +\int \frac{d \omega}{2 \pi} f_{-}(\omega) \delta \Gamma\left\{\left|u_{0}(t, \omega)\right|^{2}+\left(\Gamma_{\phi}^{2}+2 \delta E^{2}-\left(\Gamma^{2}-\delta \Gamma^{2}\right) \frac{\delta E}{\delta \Gamma} \sin \phi\right)\left|\frac{u_{p}(t, \omega)}{\Gamma_{\phi}}\right|^{2}-2 \Gamma \operatorname{Re}\left[\frac{u_{0}^{*}(t, \omega) u_{p}(t, \omega)}{\Gamma_{\phi}}\right]\right\},
\end{aligned}
$$

and the occupation difference between the two dots, $\delta n(t)=n_{1}(t)-n_{2}(t)$, is given by

$$
\begin{aligned}
\delta n(t)= & \int \frac{d \omega}{2 \pi} \operatorname{Im}\left[u_{0}^{*}(t, \omega) \frac{u_{p}(t, \omega)}{\Gamma_{\phi}}\right]\left\{\Gamma \delta E f_{+}(\omega)\right. \\
& \left.+\left[\delta \Gamma \delta E-\frac{\sin \phi}{2}\left(\Gamma^{2}-\delta \Gamma^{2}\right)\right] f_{-}(\omega)\right\} .
\end{aligned}
$$

Here $f_{ \pm}(\omega) \equiv f_{L}(\omega) \pm f_{R}(\omega)$.
On the other hand, the current passing from the left lead to the right one through dot $i$ is given by $I_{i}=I_{L i}-$ $I_{R i}$. Summing up the two currents through the two dots, we obtain the transport net current $I=\frac{1}{2}\left(I_{L}-I_{R}\right)$. Combining the current $I_{1}$ flowing from the left to the right through the first dot with the current $-I_{2}$ flowing from the right to the left through the second dot gives the circulating current $I_{\mathrm{c}}=I_{1}-I_{2}$. Explicitly, the transient net current is given by 


$$
\begin{aligned}
I(t)=\int \frac{d \omega}{2 \pi} f_{+}(\omega) & \left\{\delta \Gamma \operatorname{Re}\left(u_{0}(t, \omega)-\frac{\Gamma}{\Gamma_{\phi}} u_{p}(t, \omega)\right)-\frac{\Gamma \delta \Gamma}{2}\left[2\left|u_{0}(t, \omega)\right|^{2}+\left(\Gamma^{2}\left[\cos ^{2} \frac{\phi}{2} \cos \phi+\frac{\sin ^{2} \phi}{2}+\frac{\delta E}{\delta \Gamma} \sin \phi\right]\right.\right.\right. \\
& \left.\left.-\delta \Gamma^{2}\left[\sin ^{2} \frac{\phi}{2} \cos \phi-\frac{\sin ^{2} \phi}{2}+\frac{\delta E}{\delta \Gamma} \sin \phi\right]+\Gamma_{\phi}^{2}+\delta E^{2}\right)\left|\frac{u_{p}(t, \omega)}{\Gamma_{\phi}}\right|^{2}\right] \\
+ & \left.\left(\Gamma^{2} \delta \Gamma\left(\cos ^{2} \frac{\phi}{2}+1\right)+\delta \Gamma^{3} \sin ^{2} \frac{\phi}{2}+\frac{\Gamma^{2}-\delta \Gamma^{2}}{2} \delta E \sin \phi\right) \operatorname{Re}\left[\frac{u_{0}^{*}(t, \omega) u_{p}(t, \omega)}{\Gamma_{\phi}}\right]\right\} \\
+\int \frac{d \omega}{2 \pi} f_{-}(\omega)\{ & \operatorname{Re}\left(\Gamma u_{0}(t, \omega)-\frac{\Gamma_{\phi}^{2}+\delta E^{2}}{\Gamma_{\phi}} u_{p}(t, \omega)\right)-\frac{\delta \Gamma^{2}\left(\cos ^{2} \frac{\phi}{2}+1\right)+\Gamma^{2} \sin ^{2} \frac{\phi}{2}}{2}\left|u_{0}(t, \omega)\right|^{2} \\
& -\frac{1}{2}\left(\left[\Gamma^{2} \cos ^{2} \frac{\phi}{2}-\delta \Gamma^{2} \sin ^{2} \frac{\phi}{2}-\delta E^{2}\right] \delta \Gamma^{2} \cos ^{2} \frac{\phi}{2}-\left[\Gamma^{2} \cos ^{2} \frac{\phi}{2}-\delta \Gamma^{2} \sin ^{2} \frac{\phi}{2}+\delta E^{2}\right] \Gamma^{2} \sin ^{2} \frac{\phi}{2}\right. \\
+ & \left.\left.\delta \Gamma^{2}\left(\Gamma_{\phi}^{2}+\Gamma^{2} \sin ^{2} \phi+2 \delta E^{2}\right)\right)\left|\frac{u_{p}(t, \omega)}{\Gamma_{\phi}}\right|^{2}+2 \Gamma \delta \Gamma^{2} \operatorname{Re}\left[\frac{u_{0}^{*}(t, \omega) u_{p}(t, \omega)}{\Gamma_{\phi}}\right]\right\},
\end{aligned}
$$

where the dependencies on $\chi$ in the Green functions $\boldsymbol{u}$ and $\boldsymbol{v}$ are exactly canceled by those of the self-energy corrections $\tilde{\boldsymbol{g}}$ and $\boldsymbol{g}$ [see Eq. (12b)], leaving the current gauge independent. The transient circulating current is given by

$$
\begin{aligned}
I_{c}(t)= & \int \frac{d \omega}{2 \pi} f_{+}(\omega) \operatorname{Im}\left\{-\frac{u_{p}(t, \omega)}{\Gamma_{\phi}}\left(\Gamma^{2}-\delta \Gamma^{2}\right) \sin \phi\right. \\
& \left.+\delta \Gamma \delta E\left[2 \frac{u_{p}(t, \omega)}{\Gamma_{\phi}}-\Gamma u_{0}^{*}(t, \omega) \frac{u_{p}(t, \omega)}{\Gamma_{\phi}}\right]\right\} \\
+ & \frac{d \omega}{2 \pi} f_{-}(\omega) \operatorname{Im}\left\{u_{0}^{*}(t, \omega) \frac{u_{p}(t, \omega)}{\Gamma_{\phi}} \frac{\delta \Gamma}{2}\left(\Gamma^{2}-\delta \Gamma^{2}\right) \sin \phi\right. \\
& \left.+\delta E\left[2 \Gamma \frac{u_{p}(t, \omega)}{\Gamma_{\phi}}-\delta \Gamma^{2} u_{0}^{*}(t, \omega) \frac{u_{p}(t, \omega)}{\Gamma_{\phi}}\right]\right\} .(22)
\end{aligned}
$$

These dynamical quantities depend on the amount of the non-degeneracy $\delta E$, the coupling asymmetry $\delta \Gamma$, the magnetic flux $\phi$ and also the bias voltage applied on the leads through the particle distributions in the two electronic reservoirs. The time scales for the transient behaviors of these physical observables are determined by the factors $1 / \gamma_{\phi}^{ \pm}=2 /\left(\Gamma \pm \Gamma_{\phi}\right)$ in Eq. (16), in which the flux as well as the coupling asymmetry and the non-degeneracy play their important roles.

The symmetric and degenerate double-dot interferometer has been widely studied in the literature. This corresponds to $\delta E=\delta \Gamma=0$. Thus $\Gamma_{\phi}=\Gamma\left|\cos \frac{\phi}{2}\right|$, and the above results can be significantly simplified. Explicitly, the total occupation number in the double dot is reduced to

$$
\begin{aligned}
N(t)=\Gamma \int \frac{d \omega}{2 \pi} f_{+}(\omega)\left\{\left|u_{0}(t, \omega)\right|^{2}+\left|u_{p}(t, \omega)\right|^{2}\right. \\
\left.-2|\cos (\phi / 2)| \operatorname{Re}\left[u_{0}^{*}(t, \omega) u_{p}(t, \omega)\right]\right\},
\end{aligned}
$$

and the occupation difference between the two dots becomes

$$
\begin{aligned}
\delta n(t)= & \Gamma \sin (\phi / 2) \frac{\cos (\phi / 2)}{|\cos (\phi / 2)|} \times \\
& \int \frac{d \omega}{2 \pi} f_{-}(\omega) \operatorname{Im}\left[u_{0}^{*}(t, \omega) u_{p}(t, \omega)\right] .
\end{aligned}
$$

The transient net current is simplified to be

$$
\begin{gathered}
I(t)=\int \frac{d \omega}{2 \pi} f_{-}(\omega)\left\{-\Gamma^{2} \frac{\left|u_{0}(t, \omega)\right|^{2}-\left|u_{p}(t, \omega)\right|^{2}}{2} \sin ^{2} \frac{\phi}{2}\right. \\
\left.+\Gamma \operatorname{Re}\left[u_{0}(t, \omega)-|\cos (\phi / 2)| u_{p}(t, \omega)\right]\right\}
\end{gathered}
$$

and the circular current is given by

$$
I_{c}(t)=-\Gamma \sin (\phi / 2) \frac{\cos (\phi / 2)}{|\cos (\phi / 2)|} \int \frac{d \omega}{2 \pi} f_{+}(\omega) \operatorname{Im}\left[u_{p}(t, \omega)\right] .
$$

In general, the transient flux dependence of the physical quantities, Eqs. (19)-(22), for non-degenerate double dot with asymmetric couplings to the leads, are neither symmetric nor anti-symmetric in the flux. The complicated flux dependencies are mainly determined by the energy splitting $\delta E$. When the two quantum dots are set at degeneracy, $\delta E=0$, regardless of the coupling asymmetry and the finite applied bias, both the total occupation number and the net current become symmetric in the flux, namely, $N(\phi, t)=N(-\phi, t)$ and $I(\phi, t)=I(-\phi, t)$. In contrast, the occupation difference and the circulating current become anti-symmetric in the flux: $\delta n(\phi, t)=$ $-\delta n(-\phi, t)$ and $I_{c}(\phi, t)=-I_{c}(-\phi, t)$. However, when the degeneracy is lifted, both the symmetric and antisymmetric flux dependencies are transiently present in all these physical observables. These complicated flux dependencies can be simplified by setting an applied bias, 
$\mu_{L}=e V / 2=-\mu_{R}$. Under such a bias configuration, the terms involving $f_{-}(\omega)$ in Eqs. (20) and (22) vanish since both $\operatorname{Im}\left[u_{0}^{*}(t, \omega) u_{p}(t, \omega)\right]$ and $\operatorname{Im}\left[u_{p}(t, \omega)\right]$ are odd in $\omega$ while $f_{-}(\omega)$ is even in $\omega$. The difference in the occupations of the two dots then becomes symmetric in the flux, $\delta n(\phi, t)=\delta n(-\phi, t)$, and is proportional to $\delta E$. On the other hand, the circulating current generally contains two contributions. One is proportional to $\left(\Gamma^{2}-\delta \Gamma^{2}\right) \sin (\phi)$ and is anti-symmetric in the flux. The other contribution is proportional to $\delta \Gamma \delta E$, and is symmetric in the flux. However, we find that the second contribution decays to zero within a time scale of a few $1 / \Gamma$. After that time, the circulating current becomes anti-symmetric in the flux, proportional to $\sin (\phi)$. Applying the aforementioned bias configuration does not affect the existence of both the symmetric and the antisymmetric flux dependent components of the total occupation and the transient net current. Only in the special case of zero bias, the total occupation number becomes symmetric in the flux. The transient net current always has a non-vanishing anti-symmetric flux dependence for arbitrary values of the bias when $\delta E \neq 0$.

More interestingly, during the transient transport processes, $I(\phi, t) \neq I(-\phi, t)$ for the non-degenerate case. In other words, it transiently breaks the well-known phase rigidity at arbitrary biases. This is easily understood because during the nonequilibrium transient processes there is no time-reversal symmetry. The time-reversal symmetry is the prerequisite for phase rigidity of the linear conductance of a two-terminal device,$\frac{4.6 .7}{7}$ Only at steady state, as we show in the next section, can Eq. (21) reproduce this phase rigidity, independent of the value of $\delta E$. The on-site energy splitting thus plays a crucial role for the time-reversal symmetry breaking with respect to the flux dependency during the transient dynamics. Note also that $I(\phi, t) \neq I(-\phi, t)$ is a purely transient effect. In a steady state, the phase rigidity is preserved.

\section{COHERENCE AND PHASE RIGIDITY AT STEADY STATE}

Before studying the real-time dynamics of electronic transport in this double-dot $\mathrm{AB}$ interferometer, we deduce the steady-state results from the general transient solutions given in Sec. III, and compare them with the previous steady-state solutions obtained for a degenerate double dot coupled symmetrically to the leads.

\section{A. The general steady-state solution}

Taking the steady-state limit of Eq. (16), we have $\lim _{t \rightarrow \infty} u_{0, p}(t, \omega)=\frac{1}{2}\left(u_{-}(\omega) \pm u_{+}(\omega)\right)$ where $u_{ \pm}(\omega)=$ $\frac{1}{\gamma_{\phi}^{ \pm}-i \omega}$. Substituting these solutions into Eqs. (19)-(22), we obtain the electronic occupation and transport currents at steady state.
The total electronic occupation at steady state is reduced to

$$
\begin{array}{r}
N(\phi)=\Gamma \int_{-\infty}^{\infty} \frac{d \omega}{2 \pi} f_{+}(\omega) \frac{\left(\omega^{2}+\gamma_{\phi}^{+} \gamma_{\phi}^{-}\right)}{\left[\omega^{2}+\left(\gamma_{\phi}^{+}\right)^{2}\right]\left[\omega^{2}+\left(\gamma_{\phi}^{-}\right)^{2}\right]} \\
+\int_{-\infty}^{\infty} \frac{d \omega}{2 \pi} f_{-}(\omega)\left\{\frac{\delta \Gamma\left(\omega^{2}-\gamma_{\phi}^{+} \gamma_{\phi}^{-}\right)}{\left[\omega^{2}+\left(\gamma_{\phi}^{+}\right)^{2}\right]\left[\omega^{2}+\left(\gamma_{\phi}^{-}\right)^{2}\right]}\right. \\
\left.+\delta E \frac{\frac{\delta \Gamma \delta E}{2}-\frac{\Gamma^{2}-\delta \Gamma^{2}}{4} \sin (\phi)}{\left[\omega^{2}+\left(\gamma_{\phi}^{+}\right)^{2}\right]\left[\omega^{2}+\left(\gamma_{\phi}^{-}\right)^{2}\right]}\right\} .
\end{array}
$$

At zero bias, only the first term survives. At a finite bias, the difference of the particle distributions between the two electronic reservoirs can give an additional contribution to the total occupation when the asymmetric coupling or the non-degeneracy are present. Besides, it shows that the flux dependence of the total occupation has an anti-symmetric flux dependence only when a finite bias is applied for a non-degenerate double dot. The steady-state solution of the occupation difference is

$$
\begin{aligned}
& \delta n(\phi)= \\
& \int_{-\infty}^{\infty} \frac{d \omega}{2 \pi} \frac{\omega\left[\Gamma \delta E f_{+}(\omega)+\left(\delta \Gamma \delta E-\frac{\left(\Gamma^{2}-\delta \Gamma^{2}\right)}{2} \sin (\phi)\right) f_{-}(\omega)\right]}{\left(\omega^{2}+\left(\gamma_{\phi}^{+}\right)^{2}\right)\left(\omega^{2}+\left(\gamma_{\phi}^{-}\right)^{2}\right)} .
\end{aligned}
$$

Equation (28) shows further that one must have either an on-site energy splitting or a nonzero flux under a finite bias to generate an occupation difference between the two dots.

The general expression of the steady-state net current is

$$
I(\phi)=\int \frac{d \omega}{2 \pi}\left[f_{L}(\omega)-f_{R}(\omega)\right] \mathcal{T}(\omega, \phi),
$$

where the transmission coefficient is

$$
\mathcal{T}(\omega, \phi)=\frac{\left(\Gamma^{2}-\delta \Gamma^{2}\right)\left[\omega^{2} \cos ^{2} \frac{\phi}{2}+\left(\frac{\delta E}{2} \sin \frac{\phi}{2}\right)^{2}\right]}{\left[\omega^{2}+\left(\gamma_{\phi}^{+}\right)^{2}\right]\left[\omega^{2}+\left(\gamma_{\phi}^{-}\right)^{2}\right]} .
$$

By taking $\delta \Gamma=0$ and $\delta E=0$, Eq. (30) reproduces the results investigated in Refs. [14, 15]. Equation (29) shows that for this two-terminal device the steady-state net current (as well as its derivative with respect to the bias, i.e. the differential conductance) obey phase rigidity $I(\phi)=I(-\phi)$. The AB flux profile of the steady-state net current exhibits a phase shift of only 0 or $\pi$ with respect to all possible variations of the system parameters, including the non-degeneracy of the double dot and the asymmetry of the couplings to the leads. Correspondingly, the steady-state circulating current is given by

$$
\begin{aligned}
& I_{c}(\phi)=\left(\Gamma^{2}-\delta \Gamma^{2}\right) \times \\
& \int_{-\infty}^{\infty} \frac{d \omega}{2 \pi} \frac{\omega\left[-\frac{\Gamma}{2} \sin (\phi) f_{+}(\omega)+\left(\delta E+\frac{\delta \Gamma}{2} \sin (\phi)\right) f_{-}(\omega)\right]}{\left[\omega^{2}+\left(\gamma_{\phi}^{+}\right)^{2}\right]\left[\omega^{2}+\left(\gamma_{\phi}^{-}\right)^{2}\right]} .
\end{aligned}
$$


Both the net current and the circulating current, see Eqs. (29) and (31), are proportional to $\Gamma^{2}-\delta \Gamma^{2}$. Their $\mathrm{AB}$ oscillation amplitudes decrease upon increasing the coupling asymmetry.

\section{B. Small and large bias limits at zero temperature}

The steady-state occupation numbers and currents, Eqs. (27)-(31), are expressed in terms of integrals over the frequency. These integrals can be explicitly carried out at zero temperature with the bias configuration $\mu_{L}=e V / 2=-\mu_{R}$.

At zero temperature, the total electronic occupation is found to be

$$
\begin{aligned}
& N(\phi)= \\
& 1+\left(\delta \Gamma-\delta E \frac{\frac{\delta \Gamma \delta E}{2}-\frac{\Gamma^{2}-\delta \Gamma^{2}}{4} \sin \phi}{\Gamma \gamma_{\phi}^{+}}\right) \frac{\tan ^{-1}\left[\frac{e V / 2}{\gamma_{\phi}^{+}}\right]}{\pi \Gamma_{\phi}} \\
& -\left(\delta \Gamma-\delta E \frac{\frac{\delta \Gamma \delta E}{2}-\frac{\Gamma^{2}-\delta \Gamma^{2}}{4} \sin \phi}{\Gamma \gamma_{\phi}^{-}}\right) \frac{\tan ^{-1}\left[\frac{e V / 2}{\gamma_{\phi}^{-}}\right]}{\pi \Gamma_{\phi}} .
\end{aligned}
$$

Assuming a small or a large bias, Eq. (32) can be further simplified,

$$
\begin{aligned}
& N(\phi) \rightarrow 1+ \\
& \begin{cases}\frac{e V}{2 \pi \gamma_{\phi}^{+} \gamma_{\phi}^{-}}\left[\delta E \frac{2 \delta \Gamma \delta E-\left(\Gamma^{2}-\delta \Gamma^{2}\right) \sin \phi}{4 \gamma_{\phi}^{+} \gamma_{\phi}^{-}}-\delta \Gamma\right] & \text { if } e V \ll \Gamma \\
\frac{\delta E}{2 \Gamma} \frac{2 \delta E \delta \Gamma-\left(\Gamma^{2}-\delta \Gamma^{2}\right) \sin \phi}{\left(\Gamma^{2}-\delta \Gamma^{2}\right) \sin ^{2}(\phi / 2)+\delta E^{2}} & \text { if } e V \gg \Gamma\end{cases}
\end{aligned}
$$

Note that since Coulomb interactions have been ignored, the screening effect is altogether discarded. By setting $\delta E=0$, the total occupation at large bias becomes independent of the flux. In contrast, at small bias the total occupation is flux dependent when the coupling to the leads becomes asymmetric.

The occupation difference between the two dots at zero temperature reads

$$
\begin{aligned}
& \delta n(\phi)= \\
& \begin{cases}\frac{\delta E}{2 \pi \Gamma_{\phi}} \ln \frac{(e V / 2)^{2}+\left(\gamma_{\phi}^{-}\right)^{2}}{(e V / 2)^{2}+\left(\gamma_{\phi}^{+}\right)^{2}} & \text { if } \Gamma_{\phi} \text { is real } \\
\frac{\delta E}{\pi\left|\Gamma_{\phi}\right|}\left[\tan ^{-1} \frac{(e V)^{2}+\Gamma^{2}-\left|\Gamma_{\phi}\right|^{2}}{2 \Gamma\left|\Gamma_{\phi}\right|^{2}}-\frac{\pi}{2}\right] & \text { otherwise }\end{cases}
\end{aligned}
$$

As expected, the occupation difference is proportional to $\delta E$. The small and large bias limits are

$$
\delta n(\phi) \rightarrow \begin{cases}\frac{\delta E}{\pi \Gamma_{\phi}} \ln \frac{\gamma_{\phi}^{-}}{\gamma_{\phi}^{+}} & \text {if } e V \ll \Gamma \\ 0 & \text { if } e V \gg \Gamma\end{cases}
$$

when $\Gamma_{\phi}$ is real, and otherwise

$$
\delta n(\phi) \rightarrow\left\{\begin{array}{ll}
\frac{\delta E}{\pi\left|\Gamma_{\phi}\right|}\left[\tan ^{-1} \frac{\Gamma^{2}-\left|\Gamma_{\phi}\right|^{2}}{2 \Gamma\left|\Gamma_{\phi}\right|^{2}}-\frac{\pi}{2}\right] & \text { if } e V \ll \Gamma \\
0 & \text { if } e V \gg \Gamma
\end{array} .\right.
$$

Therefore, when a small bias is applied, the on-site energy splitting effectively causes a difference in the occupations. However, when we apply a bias much larger than the energy splitting, the energy splitting becomes ineffective in rendering the occupation difference between the two dots. The bias setting with respect to the energy splitting is thus essential for the control of the occupation difference between the two dots.

Having examined the occupations in these limits, we now turn to the currents. The steady-state net current at zero temperature is found to be

$$
\begin{aligned}
I(\phi)= & \frac{\left(\Gamma^{2}-\delta \Gamma^{2}\right)}{\pi \Gamma \Gamma_{\phi}} \\
\times & \left\{\left(\gamma_{\phi}^{+} \cos ^{2} \frac{\phi}{2}-\frac{\delta E^{2} \sin ^{2} \frac{\phi}{2}}{4 \gamma_{\phi}^{+}}\right) \tan ^{-1}\left[\frac{e V / 2}{\gamma_{\phi}^{+}}\right]\right. \\
& \left.-\left(\gamma_{\phi}^{-} \cos ^{2} \frac{\phi}{2}-\frac{\delta E^{2} \sin ^{2} \frac{\phi}{2}}{4 \gamma_{\phi}^{-}}\right) \tan ^{-1}\left[\frac{e V / 2}{\gamma_{\phi}^{-}}\right]\right\} .
\end{aligned}
$$

For small or large biases, it is further reduced to

$$
\begin{aligned}
& I(\phi) \rightarrow\left(\Gamma^{2}-\delta \Gamma^{2}\right) \\
& \quad \times\left\{\begin{array}{ll}
\frac{e V}{8 \pi\left(\gamma_{\phi}^{+} \gamma_{\phi}^{-}\right)^{2}} \delta E^{2} \sin ^{2} \frac{\phi}{2} & \text { if } e V \ll \Gamma \\
\frac{1}{2 \Gamma}\left[\cos ^{2} \frac{\phi}{2}+\frac{\delta E^{2} \sin ^{2} \frac{\phi}{2}}{\left(\Gamma^{2}-\delta \Gamma^{2}\right) \sin ^{2} \frac{\phi}{2}+\delta E^{2}}\right] & \text { if } e V \ll \Gamma
\end{array} .\right.
\end{aligned}
$$

In the small bias limit, the amplitude of the $\mathrm{AB}$ oscillation in the net current increases with the on-site energy splitting. However, under a large bias, it shows two competing oscillations, $\cos ^{2}(\phi / 2)$ and $\sin ^{2}(\phi / 2)$, which results in a sub-oscillatory pattern over the main oscillation of $\cos ^{2}(\phi / 2)$, proportional to $\delta E$.

The explicit expression for the steady-state circulating current at zero temperature is

$$
\begin{aligned}
& I_{c}(\phi)=-\frac{\Gamma^{2}-\delta \Gamma^{2}}{2 \pi} \sin \phi \\
& \quad \times \begin{cases}\frac{1}{2 \Gamma_{\phi}} \ln \frac{(e V / 2)^{2}+\left(\gamma_{\phi}^{-}\right)^{2}}{(e V / 2)^{2}+\left(\gamma_{\phi}^{+}\right)^{2}} & \text { if } \Gamma_{\phi} \text { is real } \\
\frac{1}{\left|\Gamma_{\phi}\right|}\left[\tan ^{-1} \frac{(e V)^{2}+\Gamma^{2}-\left|\Gamma_{\phi}\right|^{2}}{2 \Gamma\left|\Gamma_{\phi}\right|^{2}}-\frac{\pi}{2}\right] & \text { otherwise }\end{cases}
\end{aligned}
$$

and for small or large biases it reads

$$
I_{c}(\phi) \rightarrow \begin{cases}-\frac{\left(\Gamma^{2}-\delta \Gamma^{2}\right)}{2 \pi \Gamma_{\phi}} \sin \phi \ln \frac{\gamma_{\phi}^{-}}{\gamma_{\phi}^{+}} & \text {if } e V \ll \Gamma \\ 0 & \text { if } e V \gg \Gamma\end{cases}
$$


when $\Gamma_{\phi}$ is real, and otherwise

$$
\begin{aligned}
& I_{c}(\phi) \rightarrow \\
& \left\{\begin{array}{ll}
-\frac{\left(\Gamma^{2}-\delta \Gamma^{2}\right)}{2 \pi\left|\Gamma_{\phi}\right|} \sin \phi\left[\tan ^{-1} \frac{\Gamma^{2}-\left|\Gamma_{\phi}\right|^{2}}{2 \Gamma\left|\Gamma_{\phi}\right|^{2}}-\frac{\pi}{2}\right] & \text { if } e V \ll \Gamma \\
0 & \text { if } e V \gg \Gamma
\end{array} .\right.
\end{aligned}
$$

From the above results we find that the circulating current becomes significantly large when the bias is sufficiently small. In the opposite limit, the large bias drives the electron to flow in one direction and the circulating motion is then strongly suppressed.

It is worth noting that in the case of the degenerate double dot at zero flux, the operator $A_{-}^{\dagger} A_{-}$, where $A_{-}=\frac{1}{\sqrt{2}}\left(e^{-i \chi / 2} a_{1}-e^{i \chi / 2} a_{2}\right)$ and $\chi$ is the gauge degree of freesom, gives a constant of motion: $27\left[A_{-}^{\dagger} A_{-}, \mathcal{H}\right]=0$. When one turns on a finite flux, this symmetry is broken, and the electronic occupation is changed significantly from the value at zero flux. Indeed, if one sets $\delta E=0$ and $\phi=0$ in Eq. (15) and takes the steady-state limit, one obtains $N(\phi=0)=1 / 2+\frac{\delta \Gamma}{\pi \Gamma} \tan ^{-1}\left[\frac{e V}{2 \Gamma}\right]$ at zero temperature. However, taking the zero flux limit in Eq. (32) at degeneracy, one finds $N(\phi \rightarrow 0)=1-\frac{\delta \Gamma}{2 \Gamma}+\frac{\delta \Gamma}{\pi \Gamma} \tan ^{-1}\left[\frac{e V}{2 \Gamma}\right]$. This indicates that $N(\phi=0) \neq N(\phi \rightarrow 0)$, namely the total occupation, changes abruptly across the zero flux point. On the other hand, Eq. (20) shows that at degeneracy $\delta n(\phi=0, t)=\lim _{\phi \rightarrow 0} \delta n(\phi, t)=0$, namely the occupation difference $\delta n$ is continuous across the zero flux point. By setting first $\delta E=0$ and $\phi=0$ in Eq. (15) and then taking the steady-state limit, compared with the limit $\phi \rightarrow 0$ after the steady-state limit is taken, we find that both $I$ and $I_{c}$ are continuous as the zero flux point is crossed. Thus the abrupt change upon crossing zero flux occurs only in the total electronic occupation due to the existence of an occupation constant of motion at $\phi=0$.

The results presented in this section give the general AB flux dependence of the electronic occupation and electronic transport in the steady-state limit for the nondegenerate double dot coupled asymmetrically to the leads.

\section{REAL-TIME DYNAMICS}

Having the analytical solution for the electronic occupations and the transport currents in the double-dot $\mathrm{AB}$ interferometer for an initial empty state, we now examine the real-time dynamics of the electrons for various values of the on-site energy splitting, the coupling asymmetry as well as the externally-applied bias. For simplicity, we exploit the bias configuration, $\mu_{L}=e V / 2=-\mu_{R}$.

\section{A. Degenerate double dot with asymmetric couplings to the leads $(\delta E=0$ but $\delta \Gamma \neq 0)$}

When $\delta E=0$, one can see from Eq. (16) that the time needed to reach the steady-state limit becomes considerably longer as $\phi$ approaches zero. This is because the dominant decay factor given by $\gamma_{\phi}^{-}=$ $\frac{1}{2}\left(\Gamma-\Gamma_{\phi}\right)$ in Eq. (16) becomes smaller as $\Gamma_{\phi}=$ $\sqrt{\Gamma^{2} \cos ^{2}(\phi / 2)+\delta \Gamma^{2} \sin ^{2}(\phi / 2)}>0$ becomes larger, when $\phi$ approaches zero. However, at $\phi=0, \Gamma-\Gamma_{\phi=0}=0$ and the time to reach the steady-state limit, given by $\left(\gamma_{\phi=0}^{-}\right)^{-1}=\frac{1}{2}\left(\Gamma+\Gamma_{\phi=0}\right)^{-1}=\Gamma^{-1}$, becomes much shorter. This is because the applied magnetic flux breaks the occupation symmetry associated with the degeneracy, as we have discussed at the end of Sec. IV. As a result, the total occupation is discontinuous across the zero flux point at steady state. In the time domain, this effect is manifested as the apparent elongation of the time scale for reaching the steady-state limit at small but nonzero fluxes. The nonequilibrium occupation dynamics of the system with and without a threading magnetic flux becomes therefore significantly different.
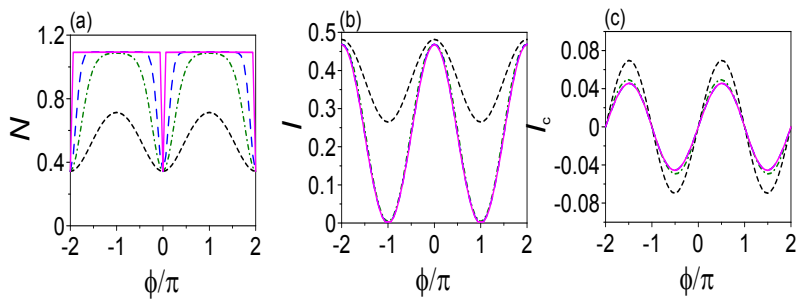

FIG. 2: Flux dependence of the occupations and currents at several different times for $\delta E=0$. The quantum dots are initially empty. The difference in the occupation numbers is not shown since it remains zero at degeneracy. The black short-dashed line is for $t=2 / \Gamma$, the green dash-dotted line is for $t=10 / \Gamma$, the blue long-dashed line is for $t=40 / \Gamma$ and $t=\infty$ is the magenta solid line. The bias is $e V=3 \Gamma$, the asymmetric coupling is $\delta \Gamma=-0.5 \Gamma$ and the temperature is $k_{B} T=\Gamma / 20$. The parameters used here are also used in other figures unless otherwise stated.

The time-dependent $\mathrm{AB}$ oscillations of the total occupation, the net current and the circulating current are shown in Figs. 2(a), (b) and (c), respectively, for the degenerate double-dot asymmetrically coupled to the leads. The flux dependencies $N(\phi, t)=N(-\phi, t)$, $I(\phi, t)=I(-\phi, t)$ as well as $I_{c}(t) \propto \sin (\phi)$ discussed in Sec. III are shown there. In Fig. 2 (a), we see that the curve for $t=40 / \Gamma$ deviates from the curve for $t=\infty$ for fluxes near zero. A discontinuity of the total occupation across the zero flux point is shown for the curve at $t=\infty$. This demonstrates the occupation symmetry breaking by the applied flux at degeneracy as discussed in Sec. IV. In Sec. IV, we have also pointed out that both the net current and the circulating current are continuous across the zero flux point. Therefore the long times needed for the occupation to reach the steady state near 
zero flux are not expected for these currents, as shown in Fig. 2 (b) and (c).

\section{B. Non-degenerate double dot with symmetric coupling to the leads $(\delta E \neq 0$ but $\delta \Gamma=0)$}

We proceed to examine the case with arbitrary on-site energy difference on the two dots coupled symmetrically to the leads, i.e. $\delta E \neq 0$ but $\delta \Gamma=0$.
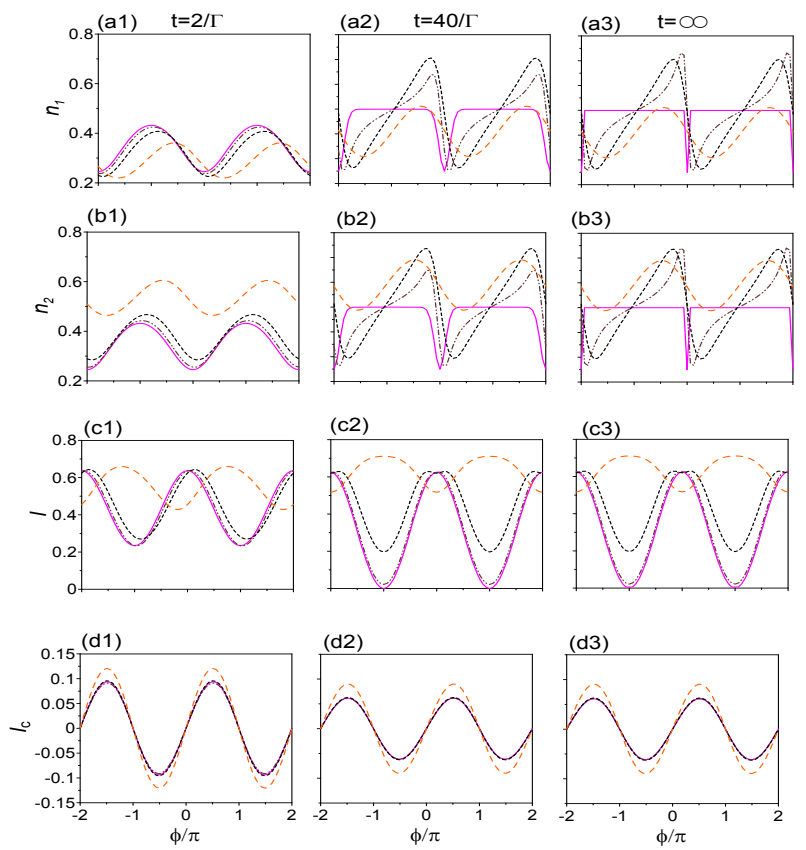

FIG. 3: Flux dependencies of the occupations and currents at several different times with various energy splittings $\delta E$. The magenta solid lines are for $\delta E=0$, the brown dash-dot-dot lines are for $\delta E=0.15 \Gamma$, the black short-dashed lines are for $\delta E=0.5 \Gamma$ and the orange long-dashed lines are for $\delta E=2 \Gamma$.

The flux dependence profiles of the occupations in each quantum dot, the net current and the circulating current are plotted in Fig. 3 for several values of the on-site energy splitting. With a small energy splitting, for example, $\delta E=0.15 \Gamma$, we can still see that the times needed for the occupations to reach the steady state at fluxes near zero are much longer than those at other values of the flux (compared Figs. 3(a2) and (b2) at $t=40 / \Gamma$ with (a3) and (b3) at $t=\infty$ ). However, the occupations at steady state are continuous across zero flux when the degeneracy of the double dot system is removed, even only slightly, as shown by the curve for $\delta E=0.15 \Gamma$ in Figs. 3(a3) and (b3). By further increasing $\delta E$, the electrons are more likely to occupy the level with the lower energy as expected, see the curves for $\delta E=2 \Gamma$ in Figs. 3(a2), (a3) and (b2), (b3). Non-symmetric flux dependencies are also observed for the occupations at $\delta E \neq 0$, as discussed in Section III.
On the other hand, upon increasing $\delta E$ to a large value (here $\delta E \sim 2 \Gamma$ ), there is a $\pi$ phase jump with respect to $\delta E=0$ in the net current, see the orange long-dashed lines in Figs. 3 (c1) to (c3). The phase jump upon changing $\delta E$ in the steady-state net current can be easily found from Eq. (29), regardless of the value of $\delta \Gamma$. More interestingly, the $\mathrm{AB}$ oscillation pattern for different $\delta E$ at time $t=2 / \Gamma$, see Fig. 3( $(\mathrm{c} 1)$, has a different phase shift as compared to those at a later time, see Figs. 3 (c2) and (c3). This shows the transiently breaking of the phase rigidity in the transient net current, as we have discussed in connection with Eq. (21). In contrast, for the case of the degenerate double dot, phase rigidity remains at all times, as shown in Fig. 2. The behavior of the circulating current in response to the change of $\delta E$ is rather simple. Its $\mathrm{AB}$ oscillations remain proportional to $\sin \phi$ at all times for different $\delta E$ with $\delta \Gamma=0$, see Figs. 3(d1) to (d3), as expected from Eq. (22).

\section{Non-degenerate double dot with the} asymmetric coupling to the leads $(\delta E \neq 0$ and $\delta \Gamma \neq 0)$

After examining the effects of the asymmetric coupling and the energy splitting separately on the electronic occupation and transport dynamics, we next study the effects of the asymmetric coupling together with a finite energy splitting
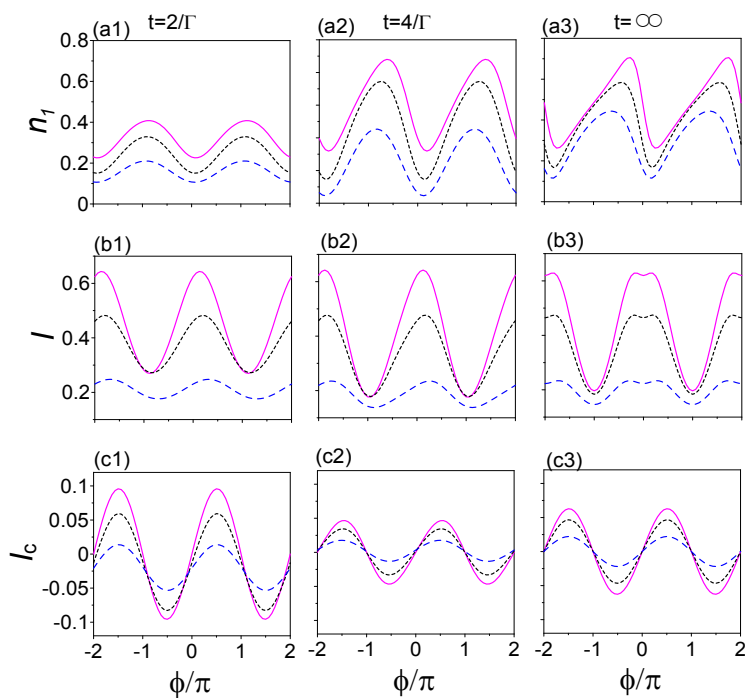

FIG. 4: The occupation number of the first dot, the net current and the circulating current as functions of flux in (a1) to (a3), (b1) to (b3) and (c1) to (c3), respectively, with $\delta E=0.5 \Gamma$. The magenta solid lines are for $\delta \Gamma=0$, the black short-dashed lines are for $\delta \Gamma=-0.5 \Gamma$ and the blue long-dashed lines are for $\delta \Gamma=-0.8 \Gamma$.

Figure 4 illustrates the results for the occupations and currents under various choices of $\delta \Gamma$ 's with $\delta E=0.5 \Gamma$. The occupation of the second quantum dot is not shown for this energy splitting since $n_{2}$ does not differ much 
from $n_{1}$. At time $t=2 / \Gamma$, the occupations for different asymmetries in the coupling have the same AB oscillation phases, see Fig. 4(a1). At long times, the differences in the $\mathrm{AB}$ oscillation phases due to different asymmetric couplings become more distinct. This demonstrates the arbitrary flux dependence of the $\mathrm{AB}$ oscillations of the total occupation with respect to the change of $\delta \Gamma$.

Figures 4(b1) to (c3) show that the amplitudes of both the transient net current and circulating current decrease upon increasing the coupling asymmetry, consistent with what we have found from Eq. (29) and Eq. (31). Inspecting the curves in Fig. 4(c1), one sees that the flux dependence of the circulating current has a small deviation from the $\sin \phi$ profile during a short time initially, but then it reaches the profile proportional to $\sin \phi$.
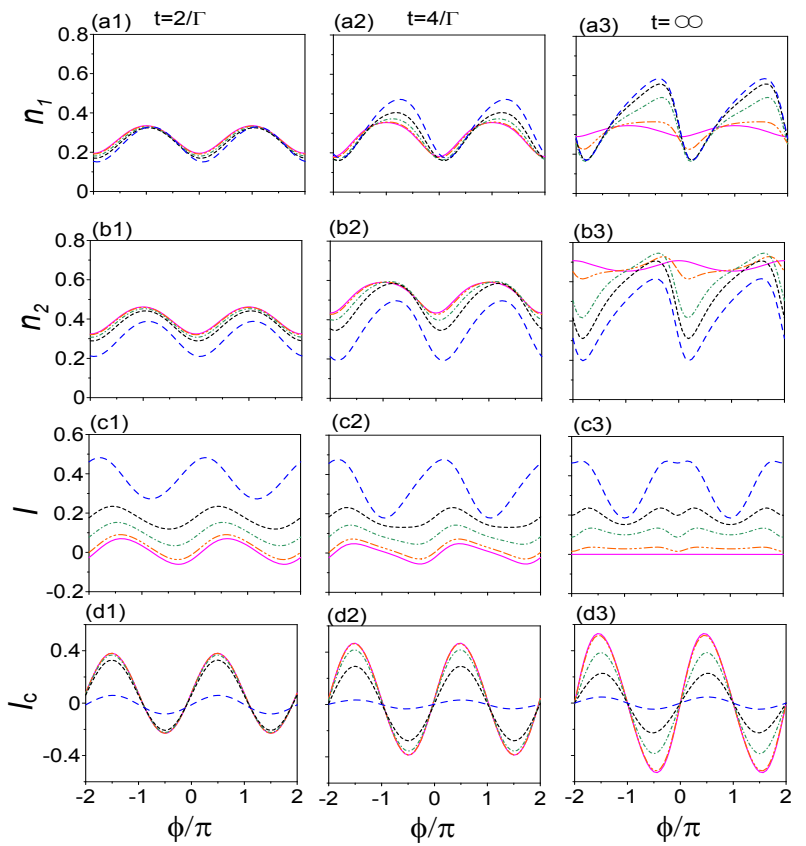

FIG. 5: The occupation numbers (a1)-(b3), the net current (c1)-(c3) and the circulating current (d1)-(d3) as functions of flux. The magenta solid lines are for $e V=0$, the orange dash-dash-dot-dot-dot lines are for $e V=0.0125 \Gamma$, the green dash-dot lines are for $e V=0.5 \Gamma$, the black short-dashed lines are for $e V=\Gamma$ and the blue long-dashed lines are for $e V=3 \Gamma$.

So far, we have examined the effects of changing the asymmetries of the system, namely, $\delta E$ and $\delta \Gamma$, at a fixed bias. We now turn to study the effects of varying the bias. The flux dependence profiles of the electronic occupations for different choices of the bias are plotted in Figs. 5(a1) to (b3), for a given energy splitting and asymmetric coupling, $\delta E=0.5 \Gamma$ and $\delta \Gamma=-0.5 \Gamma$. When the bias increases, the difference in the occupations becomes smaller [see the curves for $e V=\Gamma$ and $3 \Gamma$ in Figs. [5(a2), (a3) and (b2), (b3)]. The symmetric flux dependence of the net current is transiently broken for all biases [see Figs. 5 (c1) and (c2)]. At zero bias, the net current goes to zero at steady state, as expected, but a finite transient net current is observed [see the curve for $e V=0$ in Figs. [5.(c1) and (c2)]. At finite biases, when the net current evolves to its steady-state value, the AB oscillations develop a sub-oscillatory pattern, proportional to $\sin ^{2}(\phi / 2)$, over the main oscillation of $\cos ^{2}(\phi / 2)[$ see the curve for $e V=0.0125 \Gamma$ in Fig. 5(c3)]. The antisymmetric flux dependence is maintained for the circulating current, only the $\mathrm{AB}$ oscillation amplitudes vary in time for different biases [see Figs. 5(d1) and (d3)].

\section{CONCLUSIONS}

In this work we have explored the transient quantum dynamics of a double-quantum-dot AB interferometer using the exact solution of the master equation. We analyzed the effects of various tunable parameters of the system, namely, the splitting of the on-site energies on the double dot, the asymmetric coupling to the left and the right leads and the externally-applied bias, on the timedependent electronic occupations and the net current as well as on the circulating current, during the nonequilibrium transient processes. In the steady-state limit, we recover the results that have been extensively investigated in the literature.

With identical on-site energies on the double dot, regardless of the coupling asymmetry to the leads, we find that the total electronic occupation in the double dot and the net current are always symmetric in the flux, while the occupation difference between the two dots and the circulating current are anti-symmetric in it. We also find that the time needed for the total occupation to reach its steady-state value is much longer near zero flux, compared with the flux values away from zero. This is because there exists an occupation symmetry at zero flux, where a discontinuity cross zero flux in the total occupation is found. By breaking the degeneracy of the double dot, the phase rigidity in the net current is broken transiently at an arbitrary bias. By varying the non-degeneracy of the double dot and the coupling asymmetry to the leads, the total occupation has an arbitrary flux dependence at finite biases. The non-degenerate double dot with an asymmetric coupling to the leads makes the circulating current to slightly deviate from the anti-symmetric flux dependence initially, but it then quickly approaches the $\mathrm{AB}$ oscillations with the fully anti-symmetric flux dependence. The net current shows a sub-oscillatory pattern over the main oscillation of $\cos \phi$ at finite bias. It is also shown that a small bias causes a large circulating current whereas the net current is negligible. Thus the circulating current may provide new insights into electron coherence during the transport.

In short, the splitting of the on-site energies on the double dot and the bias configuration applied to the leads change significantly the flux dependencies of the transient electronic occupations as well as the transient transport currents. We hope that experimentally monitoring the 
transient behaviors will deepen our understanding of the electronic dynamics in quantum-dot $\mathrm{AB}$ interferometers.

\section{Acknowledgments}

This work is partially supported by the National Science Council (NSC) of ROC under Contract No. NSC-
99-2112-M-006-008-MY3. We also acknowledge support from the National Center for Theoretical Science of NSC and the High Performance Computing Facility in the National Cheng Kung University. OEW and AA acknowledge support from the Israel Science Foundation.
* Electronic address: wzhang@mail.ncku.edu.tw

† Electronic address: orawohlman@bgu.ac.il Also at Tel Aviv University, Tel Aviv 69978, Israel

¥ Electronic address: aaharonyaa@bgu.ac.il Also at Tel Aviv University, Tel Aviv 69978, Israel

1 M. Büttiker, Phys. Rev. B 46, 12485 (2992).

2 Y. Imry, Introduction to Mesoscopic Physics, $2^{\text {nd }}$ ed. (Oxford University Press, Oxford, 2002).

3 I. L. Aleiner, Ned S. Wingreen, and Y. Meir, Phys. Rev. Lett. 79, 3740 (1994).

4 A. Yacoby, M. Heiblum, D. Mahalu, and H. Shtrikman, Phys. Rev. Lett. 74, 4047 (1995).

5 R. Schuster, E. Buks, M. Heiblum, D. Mahalu, V. Umansky, and H. Shtrikman, Nature 385, 417 (1997).

6 A. Yacoby, R. Schuster, and M. Heiblum, Phys. Rev. B 53, 9583 (1996).

7 A. L. Yeyati and M. Büttiker, Phys. Rev. B 52, R14360 (1995).

8 G. Hackenbroich, and H. A. Weidenmüller, Phys. Rev. Lett. 76, 110 (1996).

9 C. Bruder, R. Fazio, and H. Schoeller, Phys. Rev. Lett. 76, 114 (1996).

10 G. Hackenbroich, Phys. Rep. 343, 463 (2001).

11 O. Entin-Wohlman, A. Aharony, Y. Imry, Y. Levinson, and A. Schiller, Phys. Rev. Lett. 88, 166801 (2002).

12 A. Aharony, O. Entin-Wohlman, B. I. Halperin, and Y. Imry, Phys. Rev. B 66, 115311 (2002).

13 A. Aharony, O. Entin-Wohlman and Y. Imry, Phys. Rev. Lett. 90, 156802 (2003).

14 B. Kubala and J. König, Phys. Rev. B 65, 245301 (2002).

15 J. König and Y. Gefen, Phys. Rev. Lett. 86, 3855 (2001); J. König and Y. Gefen, Phys. Rev. B. 65, 045316 (2002).
16 Z. T. Jiang, Q. F. Sun, X. C. Xie, and Y. Wang, Phys. Rev. Lett. 93, 076802 (2004).

17 M. Sigrist, T. Ihn, K. Ensslin, D. Loss, M. Reinwald, and W. Wegscheider, Phys. Rev. Lett. 96, 036804 (2006).

18 V. I. Puller and Y. Meir, Phys. Rev. Lett. 104, 256801 (2010).

19 M. W. Y. Tu, W. M. Zhang, and J. S. Jin, Phys. Rev. B 83, 115318 (2011).

20 M. W. Y. Tu and W. M. Zhang, Phys. Rev. B 78, 235311 (2008); M. W. Y. Tu, M. T. Lee, and W. M. Zhang, Quantum Inf. Processing (Springer) 8, 631 (2009).

21 J. S. Jin, M. W. Y. Tu, W. M. Zhang, and Y. J. Yan, New J. Phys. 12, 083013 (2010).

22 A. J. Leggett, S. Chakravarty, A. T. Dorsey, M. P. Fisher, A. Garg, and W. Zwerger, Rev. Mod. Phys. 59, 1 (1987).

${ }^{23}$ H. Haug and A.-P. Jauho, Quantum Kinetics in Transport and Optics of Semiconductors, Springer Series in Solid-State Sciences 123, 2nd Ed. (Springer-Verlag, Berlin, 2008).

24 B. Spivak and A. Zyuzin, Phys. Rev. Lett. 93, 226801 (2004).

25 D. Sánchez and M. Büttiker Phys. Rev. Lett. 93, 106802 (2004)

26 V. Puller, Y. Meir, M. Sigrist, K. Ensslin and T.Ihn, Phys. Rev. B 80, 035416 (2009).

27 Such an occupation symmetry was first found in other systems we have recently studied, H. N. Xiong, W. M. Zhang, M. W. Y. Tu and D. Braun (in preparation, 2012). Also note that though the definition of $A_{-}$involves the gauge degree of freedom $\chi$, the occupation, $\left\langle A_{-}^{\dagger} A_{-}\right\rangle$, is gauge invariant. 